\title{
Cryopreservation studies of an artificial co-culture between the cobalamin-requiring green alga Lobomonas rostrata and the bacterium Mesorhizobium loti
}

\author{
Christian J. A. Ridley ${ }^{1}$ John G. Day ${ }^{2}$ Alison G. Smith ${ }^{1}$
}

Received: 27 February 2017 / Revised and accepted: 4 September 2017/Published online: 27 September 2017

(C) The Author(s) 2017. This article is an open access publication

\begin{abstract}
Algal-bacterial co-cultures, rather than cultures of algae alone, are regarded as having the potential to enhance productivity and stability in industrial algal cultivation. As with other inocula in biotechnology, to avoid loss of production strains, it is important to develop preservation methods for the long-term storage of these cultures, and one of the most commonly used approaches is cryopreservation. However, whilst there are many reports of cryopreserved xenic algal cultures, little work has been reported on the intentional preservation of both algae and beneficial bacteria in xenic cultures. Instead, studies have focused on the development of methods to conserve the algal strain(s) present, or to avoid overgrowth of bacteria in xenic isolates during the post-thaw recovery phase. Here, we have established a co-cryopreservation method for the long-term storage of both partners in a unialgalbacterial co-culture. This is an artificial model mutualism between the alga Lobomonas rostrata and the bacterium Mesorhizobium loti, which provides vitamin $\mathrm{B}_{12}$ (cobalamin) to the alga in return for photosynthate. Using a Planer Kryo 360 controlled-rate cooler, post-thaw viability (PTV) values of $72 \%$ were obtained for the co-culture, compared to $91 \%$ for the axenic alga. The cultures were successfully revived after 6 months storage in liquid nitrogen, and continued to exhibit mutualism. Furthermore, the alga could be cryopreserved with non-symbiotic bacteria, without bacterial overgrowth occurring. It was also possible to use less
\end{abstract}

Alison G. Smith

as25@cam.ac.uk

1 Department of Plant Sciences, University of Cambridge, Cambridge CB2 3EA, UK

2 Scottish Association for Marine Science, Scottish Marine Institute, Oban PA37 1QA, UK controllable passive freezer chambers to cryopreserve the cocultures, although the PTV was lower. Finally, we demonstrated that an optimised cryopreservation method may be used to prevent the overgrowth potential of non-symbiotic, adventitious bacteria in both axenic and co-cultures of $L$. rostrata after thawing.

Keywords Cryopreservation - Algal-bacterial co-cultures · Mutualism · Bacterial overgrowth · Lobomonas rostrata . Mesorhizobium loti

\section{Introduction}

Microbial consortia are ubiquitous across nature and are now being utilised in a wide variety of biotechnological applications, such as wastewater treatment (Unnithan et al. 2013). Consortia may be more productive than axenic cultures, due to effects such as resource-use efficiency and over-yielding (in which a community as a whole produces a greater yield than any single species in the community). Microbial consortia are successfully utilised in the hydrolytic and methanogenic bacterial communities of anaerobic digestion (Grosskopf and Soyer 2014), food production (Herve-Jimenez et al. 2009) and microbial fuel cells (Nishio et al. 2013), amongst other applications. Algal-bacterial consortia are being considered for application in advanced biorefineries, aquaculture and for environmental mitigation (Ramanan et al. 2016).

Algae possess great potential for industrial biotechnology as a result of their simple cultivation, minimal growth requirements and capability to be produced on non-arable land (Greenwell et al. 2010). However, mass microalgal culture remains a relatively expensive, energetic process and commercial success has largely been restricted to a few taxa for the 
production of high-value products (Varshney et al. 2015). High volume, low-value products, such as biofuels, have so far failed to be commercially viable, due in part to productivity losses at large scale by contamination of algal cultures by adventitious organisms, such as other algae, bacteria, fungi and zooplankton. Contamination is virtually impossible to avoid even in closed photobioreactors (Day et al. 2012). The fast growth rates of bacteria, in comparison to microalgae, and the abundance of nutrients in the culture medium, provides an ideal environment for bacterial proliferation (Sue et al. 2011). It is recognised that the contamination of algal cultures by bacterial pathogens is a significant contributor to the restricted growth of commercial-scale microalgal biotechnology for low-value products (Smith and Crews 2014). However, the presence of bacteria in algal cultures can be beneficial: mutualistic bacteria are extremely important to algal ecology and have been demonstrated to provide algae with vitamins (Croft et al. 2005; Paerl et al. 2015), iron (Amin et al. 2009) and phytohormones (Amin et al. 2015). This has led to the proposal that algal-bacterial co-cultures may be a mechanism to enhance the productivity of algal cultures, whilst reducing the likelihood of culture crash by adventitious or extraneous contaminating bacteria (Shurin et al. 2013; Kouzuma and Watanabe 2015).

In order to ensure the sustainability of production and the stability of algal-bacterial consortia, or microbial consortia more generally, it is necessary to develop preservation methods for their long-term storage. It is vital that biological inocula maintain genetic, phenotypic and functional stability so that biotechnological processes may be reproducible and consistent (Stacey and Day 2014). Whilst serial sub-culture is often the standard method used for algae (Harding et al. 2004), it may be unsuitable for the long-term storage of cultures, as genotypic and phenotypic drift can occur, with the potential loss of important characteristics of the strain (Day et al. 2005). Furthermore, culture maintenance by serial sub-culture can also result in contamination by adventitious bacteria and other microorganisms due to the increased likelihood of human error associated with frequent handling. For many microorganisms used in biotechnological applications, master stock cultures are stored as lyophilised (freeze-dried) or cryopreserved samples (Stacey and Day 2014). Unfortunately, lyophilisation of eukaryotic microalgae has proved largely unsuccessful, with little or no survival for most species (Day and Brand 2005). In contrast, cryopreservation (storage at an ultra-low temperature, between -80 and $-196{ }^{\circ} \mathrm{C}$ ) is a viable option for many algal taxa (Taylor and Fletcher 1998; Day and Brand 2005). Whilst the majority of methodology development has been performed on axenic cultures, many non-axenic taxa have also been successfully cryopreserved and examples can be seen on the websites of the major algal culture collections including CCAP (Culture Collection of Algae and Protozoa, www.ccap.ac.uk) and NCMA (National Center for Marine
Algae and Microbiota, ncma.bigelow.org). Nonetheless, with the exception of an environmental consortium of mixed algae and bacteria, employed for bioremediation of a diluted effluent stream from an anaerobic digestion plant (Silkina et al. 2017), there is no report to date of a purposeful cryopreservation of a defined algal-bacterial co-culture.

In this study, we investigated whether a co-culture comprising the chlorophyte alga Lobomonas rostrata and the rhizobial bacterium Mesorhizobium loti could be successfully cryopreserved. This artificial system, first described by Kazamia et al. (2012), was set up as a model to study alga-bacterial mutualism at the molecular level, and is based on the fact that $L$. rostrata is dependent for growth on a supply of cobalamin (vitamin $\mathrm{B}_{12}$ ), which can be provided by $M$. loti in exchange for photosynthate. The interaction is characterised by a stable algal-bacterial ratio of about $30 \mathrm{M}$. loti cells per $L$. rostrata cell, and is highly regulated, with each partner's growth dependent on that of the other species (Grant et al. 2014). We also determined whether $L$. rostrata could be cryopreserved alongside non-symbiotic bacteria without the occurrence of bacterial overgrowth upon thawing. Finally, a comparison of the practicality and applicability of standard equipment for the cryopreservation of unialgal-bacterial co-cultures was performed.

\section{Methods}

\section{Cultivation methods}

Axenic Lobomonas rostrata SAG 45-1 was cultured in autotrophic TP+ medium supplemented with vitamin $\mathrm{B}_{12}$ in the form of cyanocobalamin at a concentration of $100 \mathrm{ng} \mathrm{L}^{-1}$ as described by Kazamia et al. (2012). Cultures were maintained at $25^{\circ} \mathrm{C}$ with 120 -rpm shaking and illuminated by cool white fluorescent lamps with a photon flux density of $100 \mu \mathrm{mol}$ photons $\mathrm{m}^{-1} \mathrm{~s}^{-1}$ in a 16:8 h light-dark regime. Stock cultures were maintained by transferring $1 \mathrm{~mL}$ of dense culture into $25 \mathrm{~mL}$ of fresh $\mathrm{TP}+\left(+\mathrm{B}_{12}\right)$ once every 4 weeks. Axenicity of cultures was assessed by visual inspection of cultures grown on plates, prepared by serial dilution of $1 \mathrm{~mL}$ of culture on $\mathrm{LB}$ and TY agar plates following incubation for 3 days at $28{ }^{\circ} \mathrm{C}$ (Ridley 2016).

The sequenced strain of M. loti (MAFF303099) ( Kaneko et al. 2000) was cultured in TY broth (tryptone $5 \mathrm{~g} \mathrm{~L}^{-1}$, yeast extract $3 \mathrm{~g} \mathrm{~L}^{-1}, \mathrm{CaCl}_{2} \cdot 2 \mathrm{H}_{2} \mathrm{O} 0.875 \mathrm{~g} \mathrm{~L}^{-1}$ ) at $28^{\circ} \mathrm{C}$ for 4 days. Mesorhizobium loti pre-culture aliquots $(1 \mathrm{~mL})$ were centrifuged and the biomass pellet was washed twice in sterile $1.5 \%$ $\mathrm{NaCl}$ solution to remove residual medium, before resuspension in $1 \mathrm{~mL}$ of the $\mathrm{NaCl}$ solution. Aliquots $(1 \mathrm{~mL})$ of dense L. rostrata culture were washed by centrifugation and resuspended in $1 \mathrm{~mL}$ sterile $\mathrm{TP}+$ medium, and transferred into $25 \mathrm{~mL}$ of fresh $\mathrm{TP}+$ without vitamin $\mathrm{B}_{12}$, to which $5 \mu \mathrm{L}$ of 
the washed and resuspended $M$. loti culture was added. Cocultures were maintained under the standard $L$. rostrata cultivation conditions for 7-10 days to allow the mutualism to establish and the ratio of $M$. loti to $L$. rostrata to reach between 10:1 and 30:1, which was confirmed by assessing cell densities by using a Beckman Coulter Z2 cell counter (UK), following the method developed for this co-culture by Kazamia et al. (2012). To produce artificially contaminated L rostrata cultures, two bacteria were used, Pseudomonas fluorescens (MAFF76a) and Curtobacterium flaccumfaciens, both of which were derived from non-axenic $L$. rostrata cultures (Ridley 2016). An inoculum of $1 \times 10^{5}$ cells $\mathrm{mL}^{-1}$ of each of these bacteria was transferred into L. rostrata and $L$. rostrata $+M$. loti cultures to reach a final $L$. rostrata to bacterial-contaminant ratio of 1:1. The $L$. rostrata / bacterial mix was then cryopreserved.

To test preservation of the mutualistic co-culture, thawed samples of cultures were used to inoculate $\mathrm{TP}+$ medium and grown for several days under standard conditions. Cell counts of $L$. rostrata were determined with a Coulter counter, and those for M. loti by colony-forming units after plating serial dilutions on TY agar plates.

\section{Cryopreservation protocol}

The majority of experiments performed in this study used a Planer Kryo 360 controlled-rate cooler (Planer plc, UK), to allow the precise control of parameters required to optimise the protocol. Samples with known numbers of algal cells from cultures of $L$. rostrata or $L$. rostrata $+M$. loti were pretreated before freezing by dilution $1: 1$ into $\mathrm{TP}+$ medium $\left(+\mathrm{B}_{12}\right.$ or $-\mathrm{B}_{12}$, respectively) containing different cryoprotectants/ cryoprotective agents (CPA), namely dimethyl sulphoxide (DMSO), methanol (MeOH) or glycerol, at 5 or $10 \%(v / v$ in culture medium). After addition of the cryoprotectant, samples were incubated at room temperature for $10 \mathrm{~min}$. Aliquots $(1 \mathrm{~mL})$ were then transferred to pre-labelled cryovials. A two-step cryopreservation protocol based on that established by Morris (1981), cooling at $1{ }^{\circ} \mathrm{C}$ minute ${ }^{-1}$ to $-40^{\circ} \mathrm{C}$, with the addition of automated ice-nucleation at $-5^{\circ} \mathrm{C}$ was employed. After being held for $15 \mathrm{~min}$ at $-40{ }^{\circ} \mathrm{C}$, all samples were plunged into liquid nitrogen. Non-treated samples were simply diluted 1:1 into culture medium without CPA, and then frozen under identical conditions.

Comparative cryopreservation methods were performed using a Mr. Frosty passive cooler from Nalgene (Thermo Fisher Scientific Inc., USA) in place of the Planer Kryo 360 unit. The Mr. Frosty unit was prepared as per the manufacturers' instructions, with the addition of $250 \mathrm{~mL}$ of isopropanol in the reservoir adjacent to the chamber containing cryogenic vials and was then placed in a refrigerator overnight to equilibrate at approximately $4{ }^{\circ} \mathrm{C}$. The samples were placed into the Mr. Frosty unit and stored in a $-80{ }^{\circ} \mathrm{C}$ freezer for $90 \mathrm{~min}$ to provide a nominal cooling rate of $-1{ }^{\circ} \mathrm{C}$ minute ${ }^{-1}$ to $-80{ }^{\circ} \mathrm{C}$, before plunging into liquid nitrogen. All samples were transferred to a cryostorage dewar for storage in liquid phase liquid nitrogen for at least 1 week before thawing.

\section{Thawing and recovery procedure}

Cryopreserved samples and non-cryopreserved controls were removed from liquid nitrogen and immediately placed into a $40{ }^{\circ} \mathrm{C}$ water bath until thawed. The exterior of the vials was then surface sterilised with $70 \%$ ethanol to minimise the risk of adventitious contamination. The samples were aseptically removed, followed by 10 -fold dilution into sterile TP+ medium $\left(+/-\mathrm{B}_{12}\right)$ to reduce final cryoprotectant concentration to $<0.5 \%$. To test whether light-affected post-thaw viability (PTV) of cryopreserved samples, cells were either analysed immediately or incubated in the dark at $24^{\circ} \mathrm{C}$ for $24 \mathrm{~h}$ prior to analysis.

\section{Assessment of post-thaw viability of $L$. rostrata}

Post-thaw viability (PTV) of $L$. rostrata was calculated as the percentage of viable cells after cryopreservation versus the cell counts of the samples before cryopreservation. The viability of cells was assessed using the non-toxic fluorescent vital stain 6carboxyfluorescein succinimidyl ester (CFSE) (Thermo Fisher Scientific Inc., USA), by addition of $0.4 \mu \mathrm{L}$ of a $0.6 \mu \mathrm{M}$ CFSE stock to $1 \mathrm{~mL}$ of culture, either before cryopreservation or after thawing a sample removed from liquid nitrogen. Samples were analysed by examination under epifluorescence at $400 \times$ magnification using mirror unit UMWSG2 (Olympus, Japan) and filter set 41020 (Chroma Technology Corp, USA). An additional confirmatory staining method using 3-amino-7dimethylamino-2-methylphenazine hydrochloride (neutral red) from Sigma-Aldrich (USA) was used to assess cellular damage and viability after cryopreservation. In viable algal cells, vacuoles are stained red, whilst non-viable cells lack this localisation and display a yellow/orange cytosol (Zetsche and Meysman 2012). Cells were visualised on an Olympus BX51 epifluorescence microscope equipped with phase-contrast and DIC optics. At least 50 algal cells were counted for each sample. Data were analysed using Student's $t$ test or one-way ANOVA using Microsoft Excel. A $P$ value of $<0.05$ was considered statistically significant. All error bars display standard deviation from at least three replicate experimental runs.

\section{Results}

\section{Cryopreservation of axenic $L$. rostrata and $L$. rostrata $+M$. loti co-culture}

We first tested the survival of axenic cultures of $L$. rostrata and M. loti using different CPA, as shown in the left part of 
Fig. 1. Post-thaw viability was quantified by counting viable cells stained using CFSE as described in the Methods, and expressed as a percentage of cells originally cryopreserved. For the samples with no CPA, the PTV was $11 \pm 3 \%$. Whilst the use of $5 \%$ glycerol as the CPA resulted in an increased PTV $(25 \pm 14 \%)$, this was not significant $(P=0.1)$. The use of DMSO and $\mathrm{MeOH}$ as CPA all yielded higher PTV values than the absence of CPA, or 5\% glycerol. The most effective CPA tested appeared to be $10 \% \mathrm{MeOH}$, resulting in a statistically significant increase in PTV of $65 \pm 13 \%(P<0.001)$. However, this value was statistically indistinguishable from $5 \%$ DMSO or $5 \% \mathrm{MeOH}(52 \pm 11 \%$ and $61 \pm 40 \%$, respectively). Previous cryopreservation studies have found that PTV can be enhanced by a period of dark incubation after thawing (Silkina et al. 2017). A 24-h dark incubation of axenic L. rostrata cells increased PTV significantly using both $5 \%$ $(P=0.0005)$ and $10 \% \mathrm{MeOH}(P=0.01)$ as CPA (Fig. 2).

Validation of the efficacy of the protocol was carried out with an additional vital stain using neutral red (Fig. 3). Lobomonas rostrata cells that had not been frozen contained multiple red-stained vacuoles indicative of viable cells (Fig. $3 b)$. On subjecting cells to lethal cryo-stress by plunging samples directly into liquid nitrogen, staining indicates that the intracellular structures such as the cup-shaped chloroplast visible in Fig. 3a, were disrupted with the neutral red stain diffusely visible across the cell (Fig. 3c). In contrast, these features are retained in cells that had been cryopreserved using the standardised protocol (Fig. 3d).

After demonstrating successful cryopreservation of axenic L. rostrata, the protocol was then tested on the L. rostrata + $M$. loti co-culture. To ensure that cryoprotectant efficiency was consistent between axenic L. rostrata and the cocultured algae and bacteria, the same CPAs were tested (Fig. 1, right-hand part). The optimal CPA was again found to be $10 \% \mathrm{MeOH}(94 \pm 43 \%)$, resulting in a significant increase in

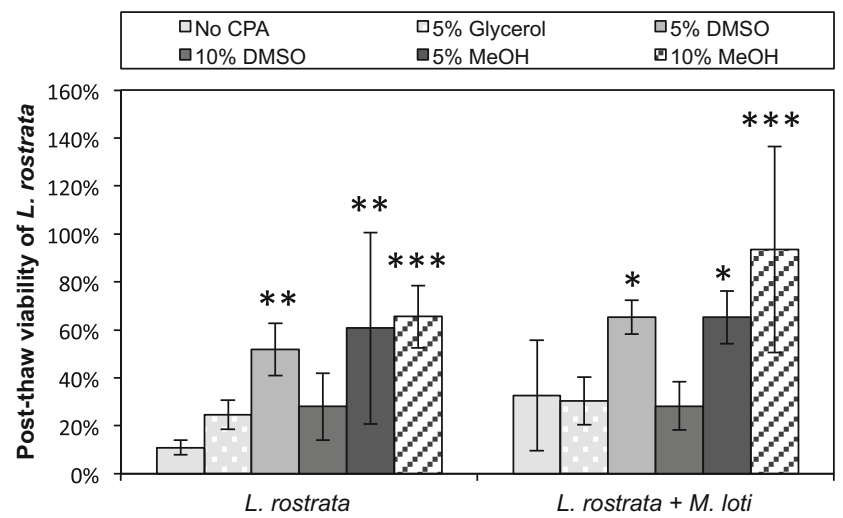

Fig. 1 Effect of cryoprotective agents on post-thaw viability (PTV). Cultures of $L$. rostrata (left) and $L$. rostrata $+M$. loti (right) were cryopreserved with different CPAs, then thawed and tested for viability by CFSE staining. Values are mean of three samples \pm standard deviation of the mean. Significance is marked by asterisks $(* P<0.05, * * P<0.01$, $* * * P<0.001)$

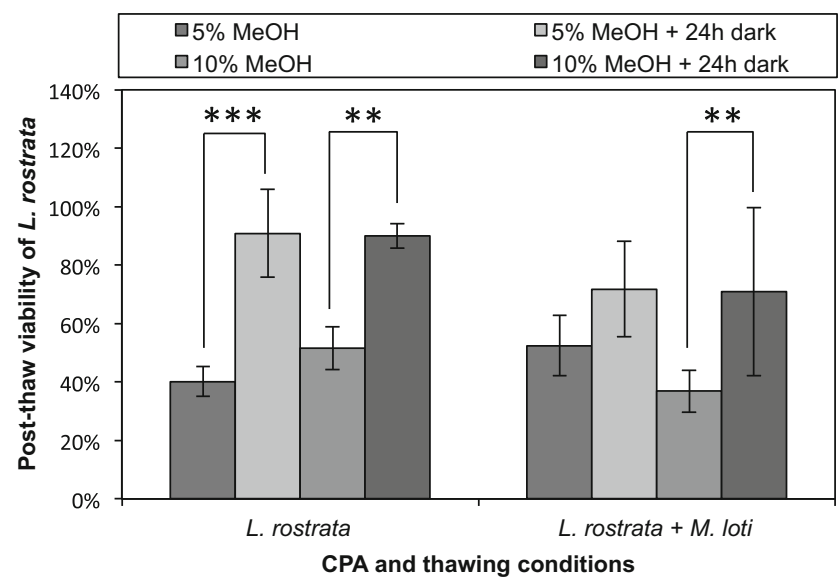

Fig. 2 Effect of a dark incubation period on PTV. Cultures of $L$. rostrata (left) and L. rostrata + M. loti (right) were cryopreserved in 5 or $10 \%$ $\mathrm{MeOH}$, and then either placed directly in the light after the removal from liquid $\mathrm{N}_{2}$, or incubated in the dark for $24 \mathrm{~h}$ after thawing before illumination. PTV was assessed as previously. Values are mean of three samples \pm standard deviation of the mean. Significance is marked by asterisks, where $* P<0.05, * * P<0.01, * * * P<0.001$

PTV compared to samples with no CPA $(P<0.001)$. There were no significant differences in PTV between axenic L. rostrata and the co-culture with M. loti. Again, a 24-h dark incubation period enhanced PTV compared to samples immediately transferred and incubated in the light $(P=0.004)$ as shown in Fig. 2 (right-hand side). Using this protocol, final post-thaw viability values of $91 \%( \pm 15 \%)$ for axenic L. rostrata and $72 \%( \pm 16 \%)$ for $L$. rostrata in co-culture with M. loti were obtained.

To determine if the mutualistic bacterium $M$. loti survived the cryopreservation process, and to investigate the maintenance and stability of the symbiotic interaction after longterm storage in liquid nitrogen, cryopreserved co-cultures were thawed after $\sim 6$ month storage in liquid nitrogen and used to inoculate fresh TP+ medium. After 24-h dark incubation, cultures were placed in the light and, as shown in Fig. 4a, L. rostrata cells survived and regrew successfully, in fact more effectively than the axenic $L$. rostrata sample (light grey line). Similarly, M. loti cells from the co-culture grew well over the time course (Fig. 4b). Moreover, the co-culture medium contained no supplementation of vitamin $\mathrm{B}_{12}$ or a carbon source, whereas it was necessary to supplement the medium for the axenic cultures with vitamin $B_{12}$. This indicates that the bacterium continued to supply this micronutrient to the alga, confirming that the mutualistic interaction had persisted. A further characteristic of this artificial co-culture is that there is regulation of the numbers of bacterial and algal cells, at an equilibrium ratio of $\sim 10-30$ to 1 (Kazamia et al. 2012; Grant et al. 2014). For the specific experiment shown here, the coculture had an initial ratio of $\sim 15 \mathrm{M}$. loti cells per $L$. rostrata cell, which rose to $78( \pm 13)$ bacteria to algae 4 days after thawing, before dropping back to the original level (Fig. 4c). 


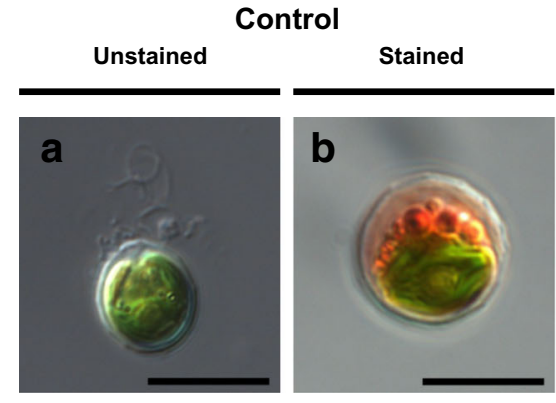

Fig. 3 DIC microscopy of neutral red-stained L. rostrata cells. a Unstained, untreated $L$. rostrata cell. b Control, non-cryoprotected, non-cooled/frozen L. rostrata cell stained with neutral red. c Non-viable cell, post-thaw with damaged vacuolar membranes. $\mathbf{d}$ Viable neutral red-

\section{Cryopreservation of xenic algal cultures containing non-symbiotic bacteria}

We also wanted to determine whether $L$. rostrata could be effectively cryopreserved when non-mutualistic bacteria were present, whilst avoiding bacterial overgrowth upon thawing, and whether the presence of the mutualistic partner had any impact on this. Two bacteria, which had previously contaminated cultures of $L$. rostrata grown under outdoor conditions (Ridley 2016), were chosen as test species. Pseudomonas fluorescens (MFAF76a) is a suspected pathogen of L. rostrata, whilst Curtobacterium flaccumfaciens is a commensal bacterium with no effect on $L$. rostrata growth (Ridley 2016). To simulate contamination, an inoculum of $1 \times 10^{5}$ cells $\mathrm{mL}^{-1}$ of each of these bacteria was added into cultures of either L. rostrata or L. rostrata $+M$. loti at a final $L$. rostrata to bacterial-contaminant ratio of $1: 1$. The L. rostrata / bacterial mix was then cryopreserved using the standardised protocol. After approximately 1 month storage under liquid nitrogen, samples were thawed and used to inoculate cultures as described above. Measurement of cell numbers (Fig. 5) revealed an initial increase in the ratio of the contaminating bacteria (P. fluorescens or C. flaccumfaciens) to $L$. rostrata in both axenic $L$. rostrata and $L$. rostrata $+M$. loti cultures in a similar pattern to that observed with $M$. loti (Fig. 4c). However, 3 days post-thaw, all ratios had reduced to be approximately equal to, or less than, pre-cryopreservation levels and no bacterial overgrowth was observed.

\section{Comparison of cooling rates}

A comparison was made between the Kryo 360 controlledrate cooler method (used up to this point in the study) and a passive freezing compartment, Mr. Frosty, to assess whether this relatively low-tech approach could be employed for the cryopreservation of the microbial consortia studied. Using the Mr. Frosty passive cooler compartment was not found to have a significant difference on the PTV of axenic L. rostrata cultures, or on the L. rostrate $+M$. loti co-culture when compared

\section{Frozen}

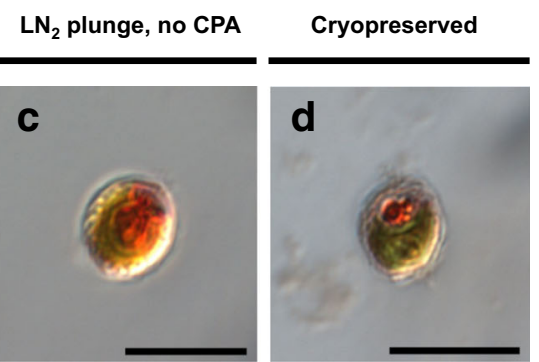

stained cells after cryopreservation employing $n 5 \% \mathrm{MeOH}$ as CPA, followed by thawing and 24-h dark incubation. Scale bar is $10 \mu \mathrm{m}$ in length. Cell size is not indicative of a change in viability

between the two different freezing methods (Fig. 6). However, a significant reduction in PTV was observed for the L. rostrata $+M$. loti co-culture $(52.6 \pm 8.6 \%)$ compared to axenic $L$. rostrata $(105.6 \pm 23.1 \%)$ when using the Mr. Frosty $(P=0.001)$.

\section{Discussion}

Whilst there have been previous studies that have investigated the implications of bacteria in the cryopreservation of algae (Amaral et al. 2013), and have cryopreserved communities of several different microbes (Kerckhof et al. 2014), to our knowledge, this study is the first to have successfully cryopreserved a mutualistic unialgal-bacterial co-culture with known characteristics (Kazamia et al. 2012) including a stable algal/ bacteria ratio. A process of methodological refinement resulted in a highly efficient cryopreservation method that produced high post-thaw viability for axenic L. rostrata and $L$. rostrata $+M$. loti co-cultures. The optimal cryoprotectant tested for both $L$. rostrata and $L$. rostrata $+M$. loti was determined to be $\mathrm{MeOH}$, in accordance with previous studies that identified $\mathrm{MeOH}$ as the more effective CPA for Chlamydomonas reinhardtii than DMSO, which is a more frequently employed cryoprotectant (Morris et al. 1979; Day and Brand 2005). Omission of a CPA, or the use of glycerol, was ineffective. The starting point for the development of this protocol was a two-step protocol developed for $C$. reinhardtii, which yielded PTV levels in our study of 72-91\%, well above the minimum PTV threshold of $60 \%$ previously recommended by Day and Fleck (2015) to ensure the successful preservation of cultures. Moreover, the characteristics of the $L$. rostrata $+M$. loti co-cultures were recovered, even after 6-month storage in liquid nitrogen. Firstly, the nutrient exchange that is the basis of the mutualism was maintained since L. rostrata continued to grow without the need for vitamin $\mathrm{B}_{12}$ supplementation (Fig. 4a). Secondly, the ratio of L. rostrata cells to $M$. loti cells in an actively growing co-culture is stable at between 1:10 and 1:30 (Kazamia et al. 2012). In this study, 


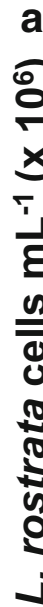

b
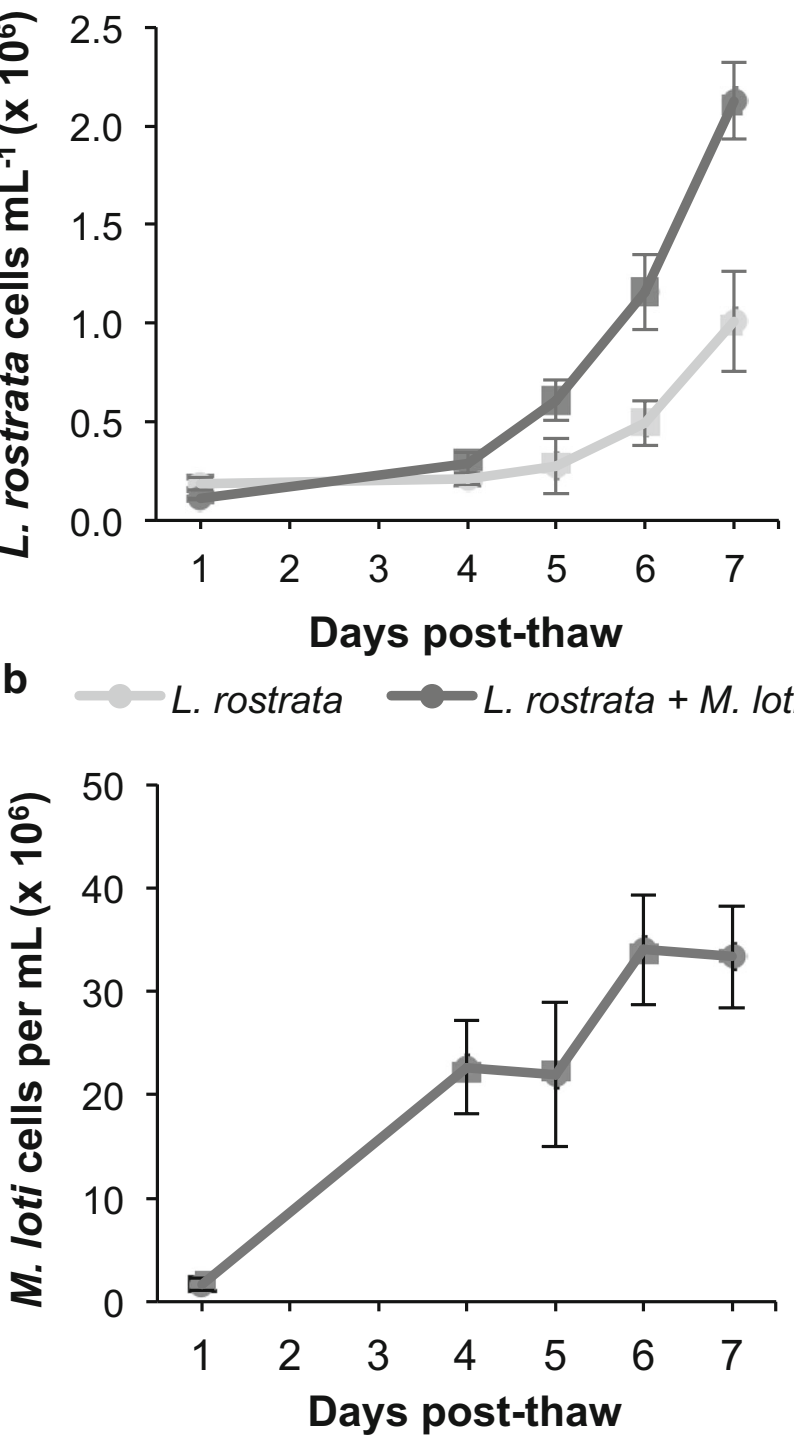

C

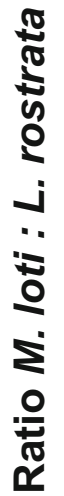
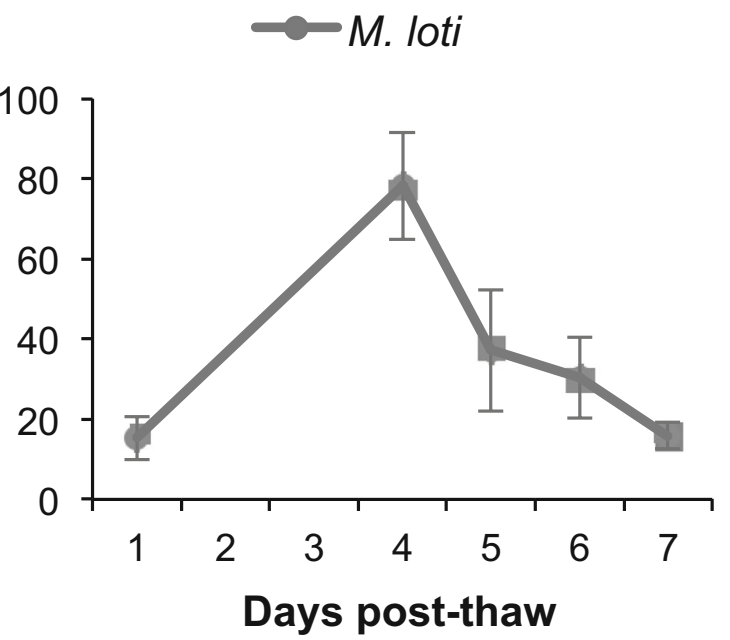

L. rostrata + M. loti
Fig. 4 Regrowth of cultures after thawing from 6 months' storage in liquid nitrogen. a Growth over 7 days of axenic L. rostrata (light grey) and L. rostrata $+M$. loti (dark grey) measured by cell counts in Coulter counter. b Growth over 7 days of $M$. loti in L. rostrata $+M$. loti coculture, measured by CFUs. $\mathbf{c}$ Ratio of numbers of $M$. loti: L. rostrata cells. Values are mean of three samples \pm standard deviation of the mean

the ratio was found to increase immediately after thawing, but decreased within expected values after several days (Fig. 4c). No overgrowth by $M$. loti, i.e. no bacterial "bloom" that results in restriction of growth or death of the algae, was observed, which suggests that co-cultures may be cryopreserved for at least several months. In theory, such stability might be maintained for decades, if not indefinitely (Grout 1995) and previous studies have demonstrated that a range of algal species remains stable after 20 years in cryostorage (Day et al.
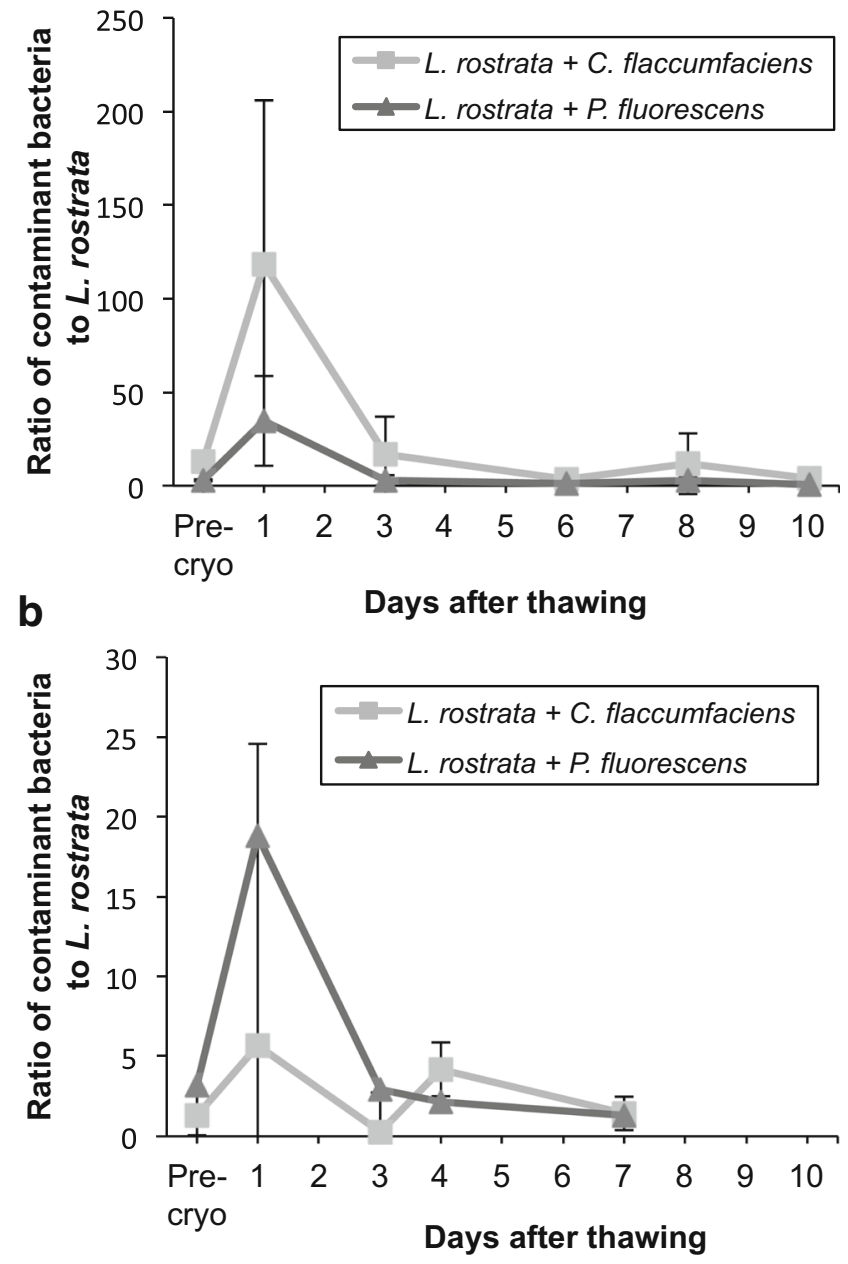

Fig. 5 Testing for overgrowth by contaminating bacteria. Cultures of a $L$. rostrata, or b $L$. rostrata $+M$. loti were inoculated with either $P$. fluorescens, or C. flaccumfaciens, then cryopreserved and thawed as previously detailed using $5 \% \mathrm{MeOH}$ as $\mathrm{CPA}$ and 24-h dark treatment. The number of contaminating bacteria was determined, and this is plotted as the ratio to L. rostrata cells. "Pre-cryo" is a control ratio calculated 1 day before cryopreservation took place. Values are mean of six samples \pm standard deviation of the mean 


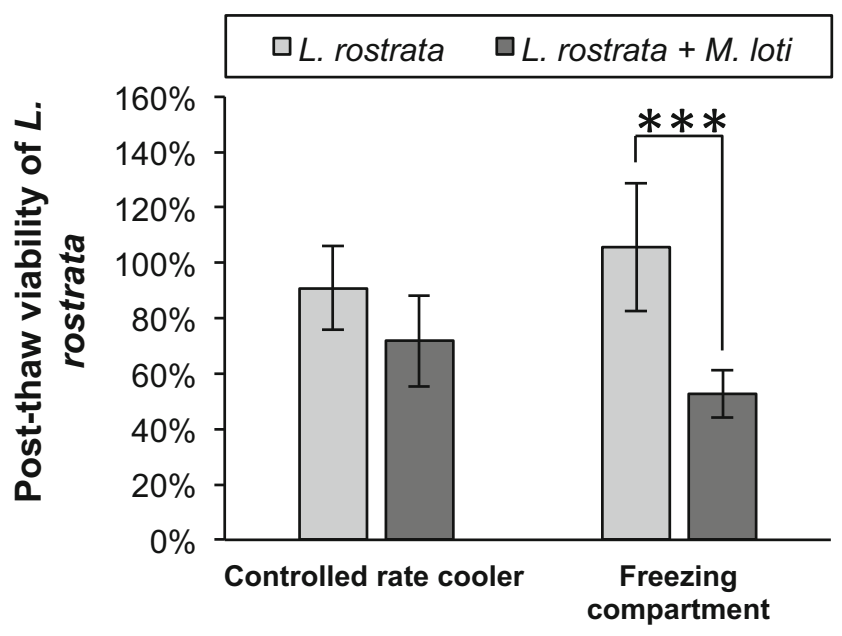

Cryopreservation method

Fig. 6 Comparison of different methods for cooling cryopreserved cultures. Cultures of either axenic L. rostrata, or L. rostrata $+M$. loti were cryopreserved using either a Planer Kryo 360 controlled-rate cooler or Mr. Frosty passive cooler, then thawed with 24-h dark treatment. PTV was assessed by CFSE staining. Values are mean of three samples \pm standard deviation of the mean. Significance is marked by asterisks, where $* P<0.05, * * P<0.01, * * * P<0.001$

1997). Additionally, we have demonstrated that algae may be cryopreserved, even with non-symbiotic bacteria, without the occurrence of bacterial overgrowth (Fig. 5). The issue of bacterial overgrowth in thawed algal cultures is a major concern in Biological Resource Centres (BRCs), as lysis of damaged cells releases additional bio-available nutrients into the recovery medium, which can stimulate a bacterial "bloom" and overgrowth of the algal culture. Heesch et al. (2012) determined that a minimum algal PTV of $25-50 \%$ was required to ensure rapid recovery of the macroalga Ectocarpus, and they included a wash step in the post-thaw process to remove the cryoprotectant and any carbon released from lysed cells. This approach has not been widely applied to microalgae as a centrifugation step is required, and this may result in additional damage to already compromised cells (Fleck 1998). In theory, antibiotics could be incorporated into the recovery medium, but in the case of CCAP this has not been routinely applied due to concerns of disrupting potential positive unialgalbacterial interactions.

The enhanced PTV observed after a period of incubation in the dark following the thawing of cultures may be related to recovery from cryo-induced damage associated with the photosynthetic apparatus. Previous studies suggest that the inclusion of a short incubation period in the dark immediately after thawing cryopreserved cells could increase PTV levels (Day and Brand 2005) and further extension of this to at least $48 \mathrm{~h}$ resulted in PTV increasing from $<1$ to $>75 \%$ for the benthic diatom Planothidium frequentissimum (Buhmann et al. 2013). Cryo-induced damage to photosynthetic apparatus in algae has also been observed in Euglena gracilis as a result of free radical production, due to metabolic uncoupling (Fleck et al. 2000). For some highly stress-tolerant algae like Haematococcus pluvialis, the presence of a coordinated antioxidant respond to oxidative stress, which is lacking in E. gracilis, may be the reason for its high cryo-tolerance (Fleck et al. 2003).

Choice of cryoprotectant may also influence the growth of bacteria post-thaw. The use of glycerol as the CPA to cryopreserve the marine red alga Gracilaria tikvahiae has been reported to be associated with bacterial overgrowth and subsequent culture contamination (van der Meer and Simpson 1984). Amaral et al. (2013) explored the implications of cryoprotectant choice on both efficacy of the cryopreservation procedure and the implications to the proliferation of partner organisms in non-axenic, mucilaginous algae and noted an increase in bacterial overgrowth when methanol, versus DMSO, was used as the CPA for a variety of microalgal species. However, in the present study, the use of methanol as CPA was not associated with bacterial overgrowth. Amaral et al. (2013) recovered, on average, slightly less than $50 \%$ of the cells after cryopreservation using DMSO or $\mathrm{MeOH}$ as $\mathrm{CPA}$, whilst in this study, up to $71 \%$ PTV was achieved in the best case. Therefore, it is possible that the high PTV we achieved prevented the overgrowth of the commensal bacteria.

A final component of this study was to determine whether a specialist controlled-rate cooler (Planer Kryo 360) was necessary for the cryopreservation of unialgal-bacterial co-cultures, or if a passive freezer compartment would be sufficient. No significant difference was observed between the PTV of $L$. rostrata and $L$. rostrata $+M$. loti using the controlled-rate cooler. Similarly, no significant differences were detected for axenic L. rostrata or the co-culture in the Planer compared to the Mr. Frosty. However, a significant decrease in PTV was identified for the co-culture when using the Mr. Frosty passive freezer compartment, compared to axenic L. rostrata. This result suggests that for axenic algal strains, the quick and easy Mr. Frosty method may be sufficient, but for the cryopreservation of unialgal-bacterial co-cultures, a controlled-rate cooler may be necessary. The reasons for such a difference are unclear, but more precise control of the cooling rate and the possibility of controlling the point at which ice-nucleation/ seeding occurs may be factors. It is possible that the two organisms differentially cryo-dehydrated in Mr. Frosty, due to the length of the treatment and the fact that the controlled-rate cooler did not go below $-40^{\circ} \mathrm{C}$, whereas the Mr. Frosty was in a $-80{ }^{\circ} \mathrm{C}$ freezer. During this phase of the cryopreservation process, cells are trapped in brine channels, which in sensitive taxa results in both physical damage and osmotic stress (Day and Fleck 2015). It is conceivable that the damage resulting from these differential stresses somehow disrupted the mutualism in the co-culture, perhaps triggering the observed overgrowth of $M$. loti upon the lysis of $L$. rostrata cells using this cooling method. 
The techniques presented in this study demonstrate the ability to cryopreserve a unialgal-bacterial co-culture. Using a well-characterised model system, we demonstrated that the mutualism, evidenced by the algal/bacteria ratio observed, remained stable after the cryopreservation process and this indicates the possibility of successfully cryopreserving more complex microbial consortia. There are many examples of algal-bacterial consortia in development that may be applied to industrial biotechnology in the near future (Do Nascimento et al. 2013; Kouzuma and Watanabe 2015; Cho et al. 2015), which may benefit from such a technique. We believe that cryopreservation is the most suitable method to ensure the availability of stable and functional inocula for industrial biotechnology, particularly if complex consortia are to be utilised.

Acknowledgements Christian Ridley was supported by a BBSRC CASE PhD studentship in collaboration with the Scottish Association for Marine Science. The authors would also like to thank Planer plc for the loan of a Kryo 360 controlled-rate cooler to complete this study.

Funding information John Day and Christian Ridley would like to acknowledge financial support from the EU for the Energetic Algae project ("EnAlgae") a Strategic Initiative of the INTERREG IVB North West Europe (NWE) Programme.

\section{Compliance with ethical standards}

Conflict of interest The authors declare that they have no conflict of interest.

Open Access This article is distributed under the terms of the Creative Commons Attribution 4.0 International License (http:// creativecommons.org/licenses/by/4.0/), which permits unrestricted use, distribution, and reproduction in any medium, provided you give appropriate credit to the original author(s) and the source, provide a link to the Creative Commons license, and indicate if changes were made.

\section{References}

Amaral R, Pereira JC, Pais AACC, Santos LMA (2013) Is axenicity crucial to cryopreserve microalgae? Cryobiology 67:312-320

Amin SA, Green DH, Hart MC, Kupper FC, Sunda WG, Carrano CJ (2009) Photolysis of iron-siderophore chelates promotes bacterialalgal mutualism. Proc Natl Acad Sci USA 106:17071-17076

Amin SA, Hmelo LR, van Tol HM, Durham BP, Carlson LT, Heal KR, Morales RL, Berthiaume CT, Parker MS, Djunaedi B, Ingalls AE, Parsek MR, Moran MA, Armbrust EV (2015) Interaction and signaling between a cosmopolitan phytoplankton and associated bacteria. Nature 522:98-101

Buhmann MT, Day JG, Kroth PG (2013) Post-cryopreservation viability of the benthic freshwater diatom Planothidium frequentissimum depends on light levels. Cryobiology 67:23-29

Cho D-H, Ramanan R, Heo J, Lee J, Kim B-H, Oh H-M, Kim H-S (2015) Enhancing microalgal biomass productivity by engineering a microalgal-bacterial community. Bioresour Technol 175:578-585

Croft MT, Lawrence AD, Raux-Deery E, Warren MJ, Smith AG (2005) Algae acquire vitamin $B_{12}$ through a symbiotic relationship with bacteria. Nature 438:90-93
Day JG, Brand JJ (2005) Cryopreservation methods for maintaining cultures. In: Andersen RA (ed) Algal Culturing Techniques. Academic Press, New York, pp 165-187

Day JG, Fleck RA (2015) Cryo-injury in algae and the implications this has to the conservation of micro-algae. Microalgae Biotechnol 1. doi:https://doi.org/10.1515/micbi-2015-0001

Day JG, Watanabe MM, Morris GJ, Fleck RA, McLellan MR (1997) Long-term viability of preserved eukaryotic algae. J Appl Phycol 9:121-127

Day JG, Benson EE, Harding K, Knowles B, Idowu M, Bremner D, Santos L, Santos F, Friedl T, Lorenz M, Lukesova A, Elster J, Lukavsky J, Herdman M, Rippka R, Hall T (2005) Cryopreservation and conservation of microalgae: the development of a pan-European scientific and biotechnological resource (the COBRA project). CryoLetters 26:231-238

Day JG, Slocombe SP, Stanley MS (2012) Overcoming biological constraints to enable the exploitation of microalgae for biofuels. Bioresour Technol 109:245-251

do Nascimento M, Dublan Mde L, Ortiz-Marquez JC, Curatti L (2013) High lipid productivity of an Ankistrodesmus-Rhizobium artificial consortium. Bioresour Technol 146:400-407

Fleck RA (1998) The assessment of cell damage and recovery in cryopreserve freshwater protists. $\mathrm{PhD}$ thesis, Abertay University, Dundee

Fleck RA, Benson EE, Bremner DH, Day JG (2000) Studies of free radical-mediated cryoinjury in the unicellular green alga Euglena gracilis using a non-destructive hydroxyl radical assay: a new approach for developing protistan cryopreservation strategies. Free Radic Res 32:157-170

Fleck RA, Benson EE, Bremner DH, Day JG (2003) Studies of antioxidant protection in freeze-tolerant and freeze-sensitive microalgae: applications in cryopreservation protocol development. CryoLetters 24:213-228

Grant MA, Kazamia E, Cicuta P, Smith AG (2014) Direct exchange of vitamin $\mathrm{B}_{12}$ is demonstrated by modelling the growth dynamics of algal-bacterial cocultures. ISME J 8:1418-1427

Greenwell HC, Laurens LML, Shields RJ, Lovitt RW, Flynn KJ (2010) Placing microalgae on the biofuels priority list: a review of the technological challenges. Interface 7:703-726

Grosskopf T, Soyer OS (2014) Synthetic microbial communities. Curr Opin Microbiol 18:72-77

Grout BWW (1995) Introduction to the in vitro preservation of plant cells, tissues and organs. In: Grout B (ed) Genetic preservation of plant cells in vitro. Springer, Berlin, pp 1-20

Harding K, Day JG, Lorenz M, Timmermann H, Friedl T, Bremner DH, Benson EE (2004) Introducing the concept and application of vitrification for the cryo-conservation of algae "a mini review". Nova Hedwigia 79:207-226

Heesch S, Day JG, Yamagishi T, Kawai H, Küpper F (2012) Cryopreservation of the model alga Ectocarpus (Phaeophyceae). CryoLetters 33:327-336

Herve-Jimenez L, Guillouard I, Guedon E, Boudebbouze S, Hols P, Monnet V, Maguin E, Rul F (2009) Postgenomic analysis of Streptococcus thermophilus cocultivated in milk with Lactobacillus delbrueckii subsp. bulgaricus: involvement of nitrogen, purine, and iron metabolism. Appl Environ Microbiol 75:2062-2073

Kaneko T, Nakamura Y, Sato S, Asamizu E, Kato T, Sasamoto S et al (2000) Complete genome structure of the nitrogen-fixing symbiotic bacterium Mesorhizobium loti. (Supplement). DNA Res 7:381-406

Kazamia E, Czesnick H, Nguyen TTV, Croft MT, Sherwood E, Sasso S, Hodson SJ, Warren MJ, Smith AG (2012) Mutualistic interactions between vitamin $\mathrm{B}_{12}$-dependent algae and heterotrophic bacteria exhibit regulation. Environ Microbiol 14:1466-1476

Kerckhof F-M, Courtens ENP, Geirnaert A, Hoefman S, Ho A, VilchezVargas R, Pieper DH, Jauregui R, Vlaeminck SE, Van de Wiele T, 
Vandamme P, Heylen K, Boon N (2014) Optimized cryopreservation of mixed microbial communities for conserved functionality and diversity. PLoS One 9:e99517

Kouzuma A, Watanabe K (2015) Exploring the potential of algae/bacteria interactions. Curr Opin Biotechnol 33:125-129

Morris GJ (1981) Cryopreservation. An introduction to cryopreservation in culture collections. Institute of Terrestrial Ecology, Cambridge $27 \mathrm{pp}$

Morris GJ, Coulson G, Clarke A (1979) The cryopreservation of Chlamydomonas. Cryobiology 16:401-410

Nishio K, Hashimoto K, Watanabe K (2013) Light/electricity conversion by defined cocultures of Chlamydomonas and Geobacter. J Biosci Bioeng 115:412-417

Paerl RW, Bertrand EM, Allen AE, Palenik B, Azam F (2015) Vitamin B1 ecophysiology of marine picoeukaryotic algae: strain-specific differences and a new role for bacteria in vitamin cycling. Limnol Oceanogr 60:215-228

Ramanan R, Kim B-H, Cho D-H, Oh H-M, Kim S-H (2016) Algaebacteria interactions: evolution, ecology and emerging applications. Biotechnol Adv 34:14-29

Ridley CJA (2016) Microbial consortia - a way forward for the commercialisation of microalgae for industrial biotechnology? $\mathrm{PhD}$ Thesis, University of Cambridge

Shurin JB, Abbott RL, Deal MS, Kwan GT, Litchfield E, McBride RC, Mandal S, Smith VH (2013) Industrial-strength ecology: trade-offs and opportunities in algal biofuel production. Ecol Lett 16:1393-1404
Silkina A, Nelson GD, Bayliss CE, Pooley CL, Day JG (2017) Bioremediation efficacy-comparison of nutrient removal from an anaerobic digest waste-based medium by an algal consortium before and after cryopreservation. J Appl Phycol 29:1331-1341

Smith VH, Crews T (2014) Applying ecological principles of crop cultivation in large-scale algal biomass production. Algal Res 4:23-34

Stacey GN, Day JG (2014) Putting cells to sleep for future science. Nat Biotechnol 32:320-322

Sue T, Obolonkin V, Griffiths H, Villas-Bôas SG (2011) An exometabolomics approach to monitoring microbial contamination in microalgal fermentation processes by using metabolic footprint analysis. Appl Environ Microbiol 77:7605-7610

Taylor R, Fletcher RL (1998) Cryopreservation of eukaryotic algae - a review of methodologies. J Appl Phycol 10:481-501

Unnithan VV, Unc A, Smith GB (2013) Mini-review: a priori considerations for bacteria-algae interactions in algal biofuel systems receiving municipal wastewaters. Algal Res 4:35-40

van der Meer JP, Simpson FJ (1984) Cryopreservation of Gracilaria tikvahiae (Rhodophyta) and other macrophytic marine algae. Phycologia 23:195-202

Varshney P, Mikulic P, Vonshak A, Beardall J, Wangikar PP (2015) Extremophilic micro-algae and their potential contribution in biotechnology. Bioresour Technol 184:363-372

Zetsche EM, Meysman FJR (2012) Dead or alive? Viability assessment of micro- and mesoplankton. J Plankton Res 34:493-509 